\title{
Effects of task difficulty on evoked gamma activity and ERPs in a visual discrimination task
}

\author{
Daniel Senkowski, Christoph S. Herrmann* \\ Max-Planck-Institute of Cognitive Neuroscience, Leipzig, Germany
}

Accepted 2 August 2002

\begin{abstract}
Objective: The present study examined oscillatory brain activity of the EEG gamma band and event-related potentials (ERPs) with relation to the difficulty of a visual discrimination task.

Methods: Three tasks with identical stimulus material were performed by 9 healthy subjects. The tasks comprised a passive control task, and an easy and a hard visual discrimination task, requiring discrimination of the color of circles. EEG was recorded from 26 electrodes. A wavelet transform based on Morlet wavelets was employed for the analysis of gamma activity.

Results: Evoked EEG gamma activity was enhanced by both discrimination tasks as compared to the passive control task. Within the two discrimination tasks, the latency of the evoked gamma peak was delayed for the harder task. Higher amplitudes of the ERP components N170 and P300 were found in both discrimination tasks as compared to the passive task. The N2b, which showed a maximum activation at about $260 \mathrm{~ms}$, was increased in the hard discrimination task as compared to the easy discrimination task.

Conclusions: Our results indicate that early evoked gamma activity and N2b are related to the difficulty of visual discrimination processes. A delayed gamma activity in the hard task indicated a longer duration of stimulus processing, whereas the amplitude of the N2b directly indicates the level of task difficulty. (C) 2002 Elsevier Science Ireland Ltd. All rights reserved.
\end{abstract}

Keywords: Event-related potential; P3; Gamma; $40 \mathrm{~Hz}$; Difficulty; Color discrimination

\section{Introduction}

The EEG gamma band (range about 20-80 Hz) has been reported to be closely related to binding processes (Engel and Singer, 2001; Tallon et al., 1995), priming effects (Elliott et al., 2000), affective processing (Keil et al., 2001; Müller et al., 1999), visual attention (Fries et al., 2001; Müller et al., 2000) and memory processing (Fell et al., 2001; Herrmann and Mecklinger, 2001; Tallon-Baudry et al., 1998). One aspect which has not yet been examined is the relation between EEG gamma activity and task difficulty. Some evidence for a relation between EEG gamma activity and task difficulty came from studies which compared EEG gamma activity for target and non-target stimuli in visual discrimination tasks. Evoked gamma activity in visual discrimination tasks was found to be higher in targets than in non-targets (Herrmann et al., 1999; Herr-

* Corresponding author. Max-Planck-Institute of Cognitive Neuroscience, Postfach 500 355, 04303 Leipzig, Germany. Tel.: +49341-9940-250; fax: +49-341-9940-204.

E-mail address: herrmann@cns.mpg.de (C.S. Herrmann). mann and Mecklinger, 2000). Longer reaction times (RTs) and higher error rates for targets as compared to non-targets indicate that targets are more difficult to discriminate than non-targets. However, targets in previous experiments were compared with non-targets. Therefore, the increased gamma activity could also have been related to the infrequent response to the target stimulus. Further evidence for a relation between gamma activity and difficulty resulted from memory tasks which showed that EEG gamma activity is positively related to memory load (De Pascalis and Ray, 1998). So far no study has explicitly examined the relation between difficulty in visual discrimination processes and EEG gamma activity.

The relation between event-related potentials (ERPs) and task difficulty has been investigated in various studies. Task difficulty has been mainly associated with the ERP components N1, N2 and P3. With regard to the latency of the N1 in visual discrimination tasks we will refer to the N1 component as N170. Ritter et al. $(1982,1988)$ found an increased negative deflection in the time interval of the N170 and N2 component in a choice-RT task as compared to simple-RT tasks. The N2 has also been associated with discrimination processes (Breton et al., 1988; Ritter et al., 1983). Smid et 
al. (1999) compared the latencies of the posterior N2 component $(\mathrm{N} 2 \mathrm{~b})$ in an easy and a hard color discrimination task. The latency of the N2b was delayed when color was hard to discriminate compared with when it was easy to discriminate. The $\mathrm{N} 2 \mathrm{~b}$, which has its maximum over the vertex (Näätänen et al., 1978), seems to be a marker of the intensity of discrimination processes. Another ERP component which has been associated with discrimination tasks is the P3 (Mecklinger et al., 1998). Most studies which investigated the visual or auditory evoked P3 found a delay of latency and a decrease of amplitude for more difficult discrimination processes, especially when comparing target stimuli with non-target stimuli (Polich, 1987; Palmer et al., 1994; Hoffman et al., 1985). The P3 latency and amplitude effects were found at frontal and posterior electrode sites (Comerchero and Polich, 1999).

In the present study we examined ERPs and EEG gamma activity in 3 tasks with different levels of difficulty. In all tasks the identical color stimuli were used as stimulus material. Gamma activity and ERPs of the same non-target stimulus were compared between all tasks. By comparing the same stimulus over all tasks we controlled for the influence of different factors which are related to EEG gamma activity, such as motor responses (Crone et al., 1998) and differences in the processing of different colors (Krüger et al., 2002). Possible differences in EEG gamma activity and ERPs are therefore mainly attributable to the difficulty of the visual discrimination task.

\section{Methods}

The experiment consists of 3 different tasks. All tasks employed a red circle and two green circles as stimulus material. Even though the luminances of all colors were equal, we will refer to the two green stimuli as 'light green' and 'dark green' to differentiate them verbally. The circles were presented 100 times each, in random order. In the first task (passive task) subjects were instructed to keep their eyes still and watch the stimuli without any task. An easy and a hard discrimination task followed the passive task. The order of the two discrimination tasks was randomized across subjects. In the easy task subjects were instructed to respond with a button press of their right thumb to the red circle and with a button press of their left thumb to both green circles. In the hard task subjects were instructed to respond with a button press of their right thumb to the light green circle and with a button press of their left thumb to the other two circles. In contrast to the easy task, subjects had to discriminate the two similar green circles in the hard task. RTs and EEG data were recorded during the tasks. The focus of the EEG and wavelet analysis was the comparison of the dark green circle across all 3 tasks since it served as non-target in all tasks. The response requirements and frequency of this stimulus were equal in both discrimination tasks.

\subsection{Subjects}

Fifteen subjects (age 23.4 \pm 2.0 years, 8 female) participated in the experiment. Six of the subjects had to be rejected from the analysis because their EEG data failed our criterion of at least 50 valid trials for each condition (see below). The remaining 9 subjects (age 23.4 \pm 1.7 years, 6 female) were included in the data analysis. All subjects were right-handed and had normal or correctedto-normal vision. They showed no signs of neurological or psychiatric disorder and all gave written informed consent to participate in the study.

\subsection{Stimuli}

Three circles with different colors were used as stimulus material. Circles were presented separately. The size of the circles was $6.5^{\circ}$ of visual angle. All circles had a luminance of about $4.5 \mathrm{~cd} / \mathrm{m}^{2}$ and were presented on a white background with a luminance of about $53 \mathrm{~cd} / \mathrm{m}^{2}$. According to the Commission International d'Eclairage (CIE) the colors had the following $X, Y$ coordinates: red $(X=0.584$, $Y=0.345)$, light green $(X=0.291, Y=0.536)$ and dark green $(X=0.257, Y=0.468)$. CIE coordinates and luminances were measured with a Minolta Chroma Meter CS100. Stimuli were presented for $800 \mathrm{~ms}$ with randomized inter-stimulus intervals ranging from 1150 to $1350 \mathrm{~ms}$. In the two discrimination tasks a feedback 'right' or 'wrong' was presented for $400 \mathrm{~ms}$ at the center of the screen succeeding each trial.

\subsection{Data acquisition}

The EEG was recorded with NeuroScan amplifiers using 26 tin electrodes mounted in an elastic cap. Electrodes were placed according to the International 10-10 system. The ground and reference electrode were placed near the left mastoid (M1). Electrode impedance was kept below $5 \mathrm{k} \Omega$. Horizontal and vertical electrooculogram (EOG) recordings were registered with 4 additional electrodes. Data were sampled at $500 \mathrm{~Hz}$ and analog-filtered with a $0.05 \mathrm{~Hz}$ high-pass and a $100 \mathrm{~Hz}$ low-pass filter. Data were further filtered with a digital $0.5 \mathrm{~Hz}$ high-pass filter before analyzing. An additional digital $20 \mathrm{~Hz}$ low-pass filter was applied before displaying the ERP data. Averaging epochs for ERP and EEG gamma activity lasted from $100 \mathrm{~ms}$ before to 800 ms after stimulus onset. For artifact suppression, trials were automatically excluded from averaging, if the standard deviation within a moving $200 \mathrm{~ms}$ time interval exceeded $30 \mu \mathrm{V}$ in any one of the channels. After the automatic artifact rejection all trials were visually inspected and rejected if eye movement artifacts or electrode drifts were visible. We set a criterion of at least 50 valid trials per condition for data analysis. 


\subsection{Data analysis}

Selected electrode sites were pooled to 4 topographical regions of interest (ROIs) to avoid a loss of statistical power that is inherent when repeated measures ANOVAs are used to quantify multi-channel EEG data. The left anterior region (LAR) was comprised of electrodes FP1, FC3, F3 and F7 while the left posterior region (LPR) included electrodes P3, O1, CP5 and P7. Regions over the right hemisphere included the homologous electrodes. For statistical analyses, ERP amplitudes were pooled across the electrodes in each of the ROIs. Fig. 4 shows ERPs of the standard dark green circle in the 3 tasks. In all tasks, a N170 was evoked around $170 \mathrm{~ms}$ after stimulus onset. In the two discrimination tasks an additional posteriorly pronounced negative ERP deflection at about $260 \mathrm{~ms}$ was evoked, the so called $\mathrm{N} 2 \mathrm{~b}$ component. Targets in both discrimination tasks also evoked a target P3. Latencies of P3 were about $350 \mathrm{~ms}$ in the easy and $550 \mathrm{~ms}$ in the hard task (see Fig. 5). For data analysis we defined ERP components as mean amplitudes in the following time intervals: $150-190 \mathrm{~ms}$ (N170), 240$280 \mathrm{~ms}$ (N2b), 300-500 ms (early P3) and 500-700 ms (late $\mathrm{P} 3)$. For the dark green stimulus, which served as standard in all 3 tasks, ANOVAs were computed for these time inter- vals comprising factors task and ROI. A further ANOVA investigated differences between the two target stimuli for the two discrimination tasks.

For the interpretation of gamma activity it is important to distinguish whether the oscillations occur phase-locked to a stimulus (evoked activity) or with variable phase relative to a stimulus (induced activity). For the analysis of gamma activity, a wavelet transform based on Morlet wavelets was employed. The details of the wavelet transform have been explicitly described in a previous article (Herrmann et al., 1999). According to Galambos (1992), at least 3 types of gamma activity can be differentiated: 'spontaneous', 'induced' and 'evoked'. The 'spontaneous' gamma activity is completely uncorrelated with the experimental setting. This activity is probably due to neuronal processes that do not relate to the task at hand. 'Spontaneous' gamma activity usually cancels out completely if an average ERP is computed across enough trial repetitions. For this reason, we do not focus the EEG gamma analysis on this type of activity. In contrast to the 'spontaneous' gamma activity, the 'induced' and 'evoked' gamma activity are related to the onset of an experimental condition. Fig. 1 shows the differences between induced and evoked gamma activity.

To differentiate between evoked and induced activity,

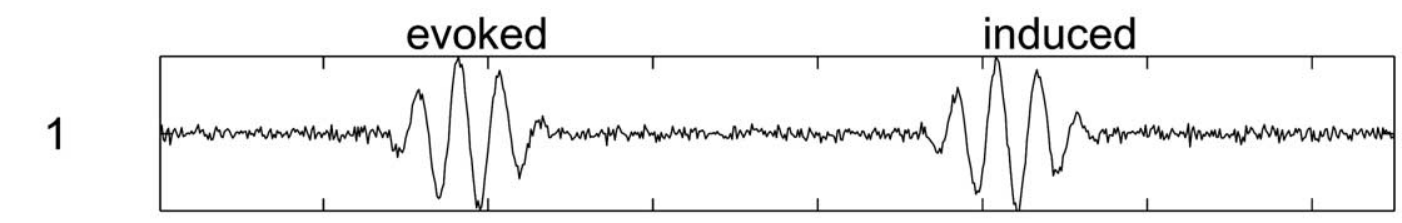

2
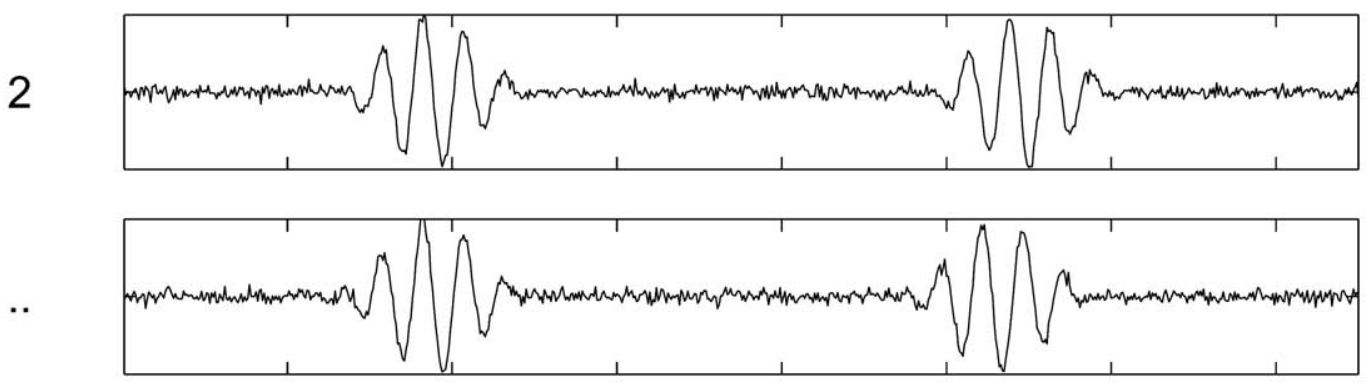

10

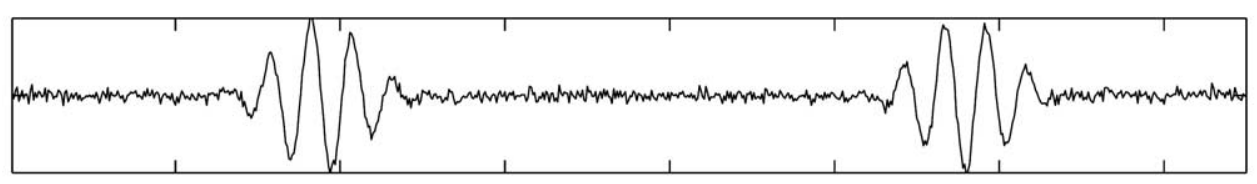

avg

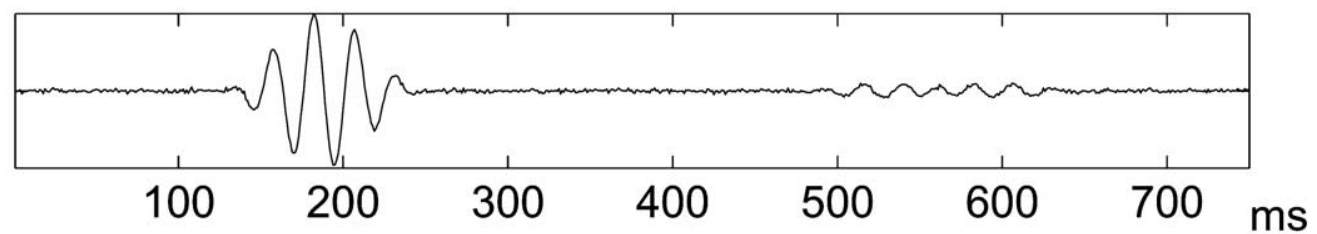

Fig. 1. Oscillations which occur at the same latency after stimulus onset with the same phase relative to stimulus onset in multiple trials (rows 1-4) are considered evoked by the stimulus (left). Evoked activity appears in the average (bottom row). Oscillations with latency or phase jitter relative to stimulus onset (right) are considered to be induced by the stimulus. Induced oscillations are cancelled out in the average. From Herrmann and Knight (2001, p. 471), with permission of the authors. 
each subject's ERP was transformed to yield evoked gamma activity and averages of transforms of single epochs were computed to yield induced activity. The frequency of gamma activity used for the wavelet analysis was individually adapted via the time-frequency plane of the $\mathrm{O} 1$ and $\mathrm{O} 2$ electrodes (Fig. 2) in response to the red circle in the easy task. The individual gamma activity was defined as the highest activation in a frequency range of about $20-80 \mathrm{~Hz}$ in a time range of about 60-200 ms. Using this definition the individually adapted gamma activity ranged from 25 to 45 Hz. In analogy to previous studies of Herrmann and Mecklinger (2001) we analyzed gamma band activity evoked by the dark green standard stimulus in an early (60-120 ms) and in a late time interval (150-250 ms) by means of an ANOVA using the same factors as for the ERP data. A further ANOVA investigated the differences between the two target stimuli. In order to examine the relations between the latencies of the EEG gamma activity and the P3 component of the ERPs we calculated a Pearson correlation coefficient.

\section{Results}

\subsection{Behavioral data}

Mean RTs and error rates of the easy and the hard discrimination task are shown in Fig. 3. A comparison of RTs between the standard dark green stimulus in the hard (570 $\mathrm{ms})$ and the easy (408 ms) task yielded longer RTs in the hard task $(F(1,8)=78.9, P<0.0001)$. The same effect was found for the targets in the two tasks. RTs of the target circle (light green) in the hard task $(614 \mathrm{~ms})$ were significantly longer as compared to the target circle (red) in the easy task
$(428 \mathrm{~ms} ; F(1,8)=117, P<0.0001)$. The delay of RTs in the hard task indicates longer stimulus processing.

At the next step the error rate of the two discrimination tasks was analyzed. Thereby, a higher error rate was found in the hard $(8.8 \%)$ as compared to the easy task $(2.0 \%)$. Comparing the error rate of the standard circle (dark green) in the two tasks yielded a significantly higher error rate in the hard $(9.8 \%)$ as compared to the easy task $(1.1 \%$; $F(1,8)=17.50, P<0.005)$. A higher error rate was also found for the target in the hard $(15.0 \%)$ as compared to the target in the easy task $(3.3 \% ; F(1,8)=22.72, P<0.005)$.

Longer RTs and higher error rates indicated that the hard task was in fact harder to perform than the easy task.

\subsection{ERP responses}

An ANOVA of N170 amplitudes in response to the standard dark green circle yielded a significant main effect of task $(F(2,16)=10.25, P<0.005)$, indicating larger amplitudes for the easy $(-1.51 \mu \mathrm{V})$ and the hard $(-1.78 \mu \mathrm{V})$ discrimination tasks as compared to the passive task $(0.02$ $\mu \mathrm{V}$; Fig. 4). A significant task $\times$ ROI interaction revealed that this effect is pronounced at posterior ROIs $(F(6,48)=3.82, P<0.05)$. Post-hoc comparisons showed no differences between the dark green circle in the easy and in the hard task $(F(1,8)=0.35, P<0.6)$. However, compared with the control condition higher N170 amplitudes were found for the easy $(F(1,8)=10.89, P<0.05)$ and the hard task $(F(1,8)=24.84, P<0.005)$, indicating that the $\mathrm{N} 170$ has a generally higher amplitude when performing a discrimination task, but is not associated with the level of difficulty. Amplitudes in the time interval of the $\mathrm{N} 2 \mathrm{~b}$ also differed between the 3 tasks $(F(2,16)=5.06, \quad P<0.05)$. Furthermore, a significant

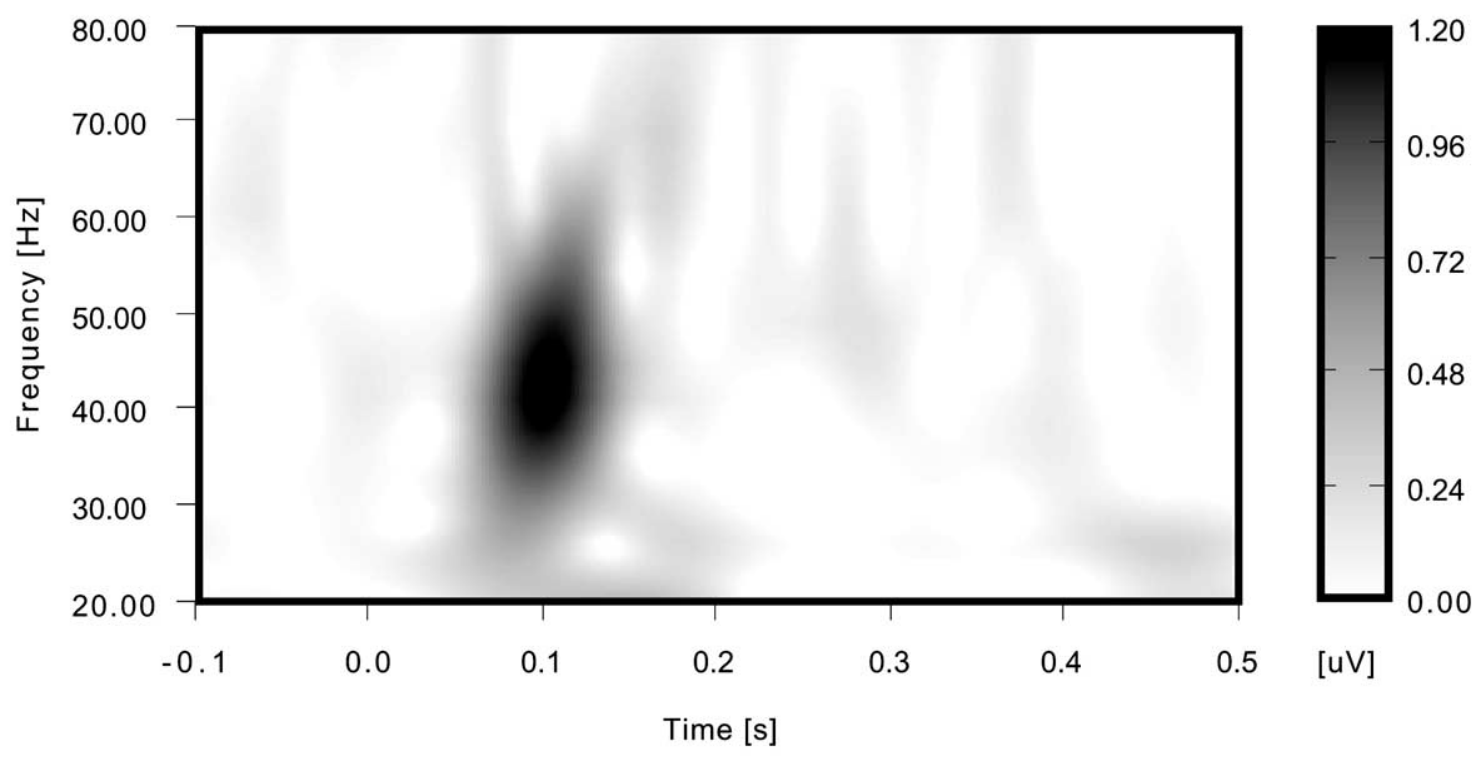

Fig. 2. Time-frequency plane of the electrode 'O2' (in one subject). After $100 \mathrm{~ms}$ an early peak of gamma activity was evoked at a frequency of about $43 \mathrm{~Hz}$. 


\section{reaction times}
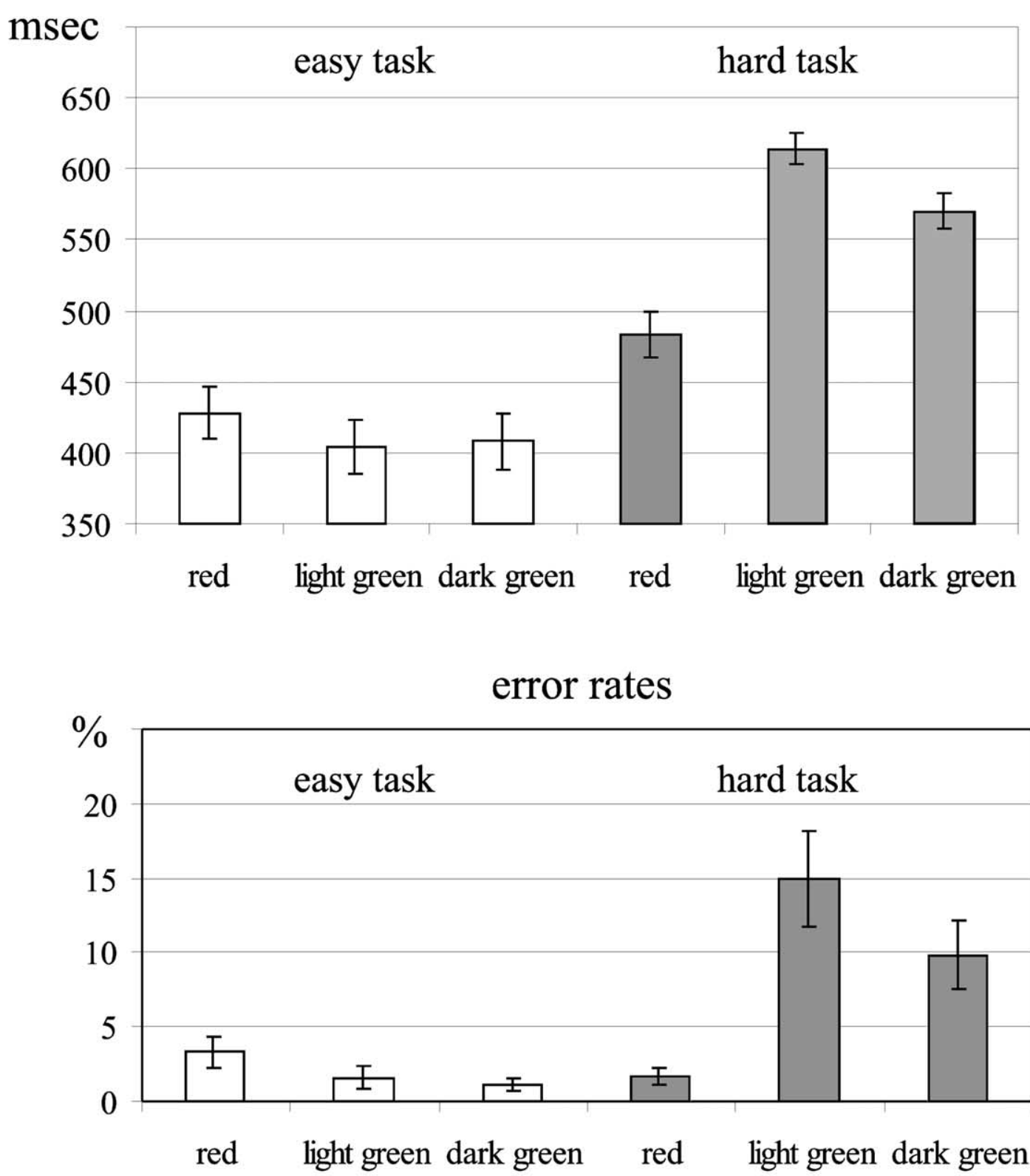

Fig. 3. Mean RTs and error rates in the easy and hard discrimination tasks. The upper figure shows RTs with standard error of the mean. The lower figure shows error rates with standard error of the mean. Notice higher error rates and longer RTs for the target stimulus (red circle in the easy task, light green circle in the hard task) and dark green standard stimulus in the hard task.

task $\times$ ROI interaction was found for $\mathrm{N} 2 \mathrm{~b}$ amplitudes $(F(6,48)=5.04, P<0.05)$, indicating that $\mathrm{N} 2 \mathrm{~b}$ effects were pronounced at posterior ROIs. A post-hoc comparison for anterior and posterior regions of the easy and the hard task revealed a significantly enhanced $\mathrm{N} 2 \mathrm{~b}$ amplitude in the hard task for posterior regions $(F(1,8)=15.65, P<0.005)$ but not for anterior regions $(F(1,8)=0.03, P<0.9)$. Amplitude differences of the standard dark green circle were also found in the time interval of the early $\mathrm{P} 3$ $(F(2,16)=9.49, P<0.005)$. As for the $\mathrm{N} 2 \mathrm{~b}$, a significant task $\times$ ROI interaction was found $(F(3,24)=6.45$,
$P<0.05)$. This interaction emerges due to the fact that only posterior P3 amplitudes were enhanced in the easy $(2.69 \mu \mathrm{V})$ as compared to the hard task $(1.25 \mu \mathrm{V}$; $F(1,8)=10.71, P<0.05)$, whereas no differences between the anterior P3 amplitudes were found $(F(1,8)=0.82$, $P<0.4$ ). For the time interval of the late P3 component no significant differences were observed for the standard dark green circle between the 3 tasks $(F(2,16)=2.81$, $P<0.1)$.

A comparison of the target $\mathrm{P} 3$ for the early $\mathrm{P} 3$ time interval between the easy and the hard task (Fig. 5) revealed 

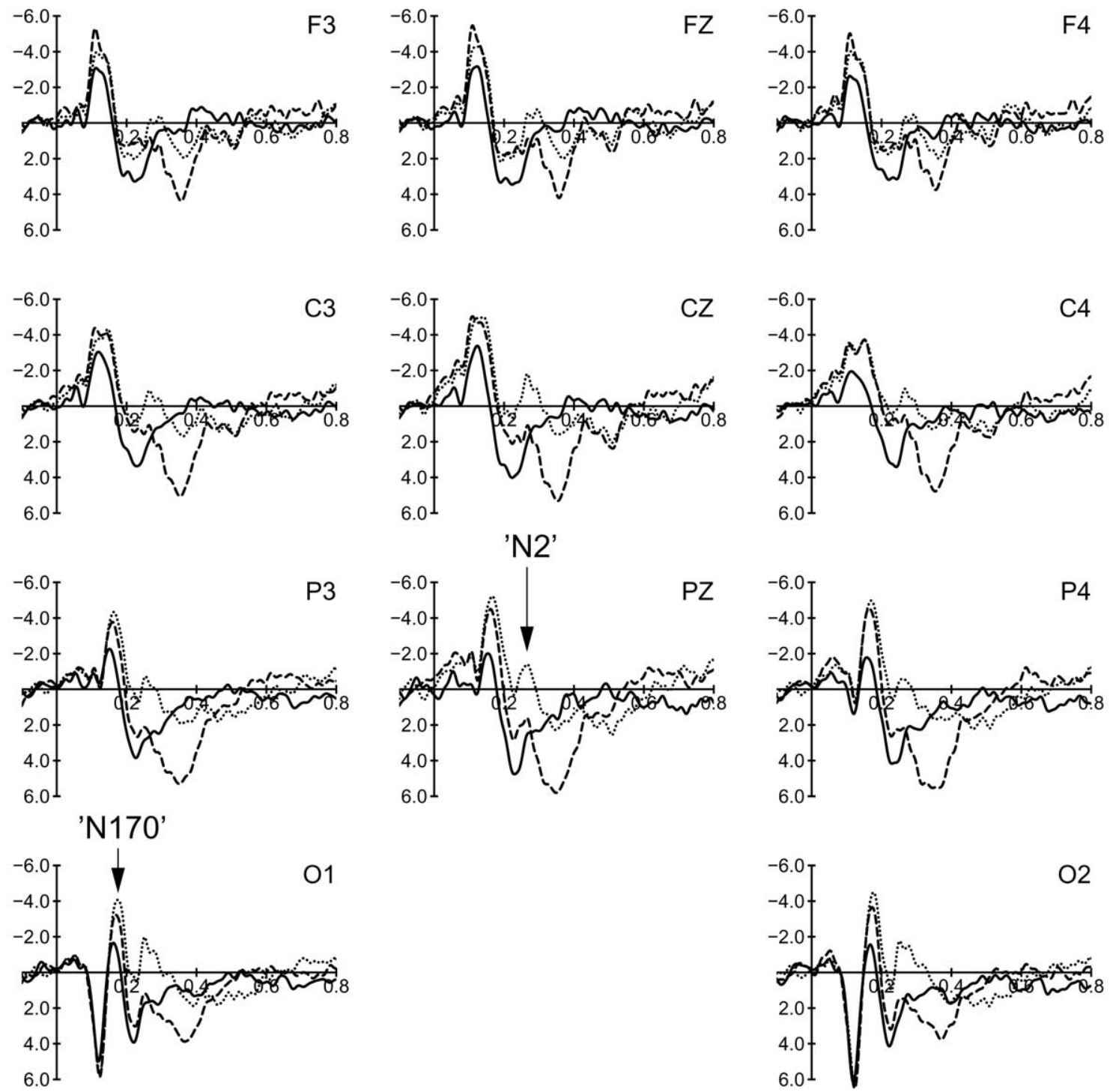

Fig. 4. ERPs averaged across 9 subjects in response to the dark green standard stimulus in the control task (solid), easy task (dashed) and hard task (dotted).

higher amplitudes for the easy $(0.93 \mu \mathrm{V})$ as compared to the hard task $(-0.19 \mu \mathrm{V} ; F(1,8)=21.39, P<0.005)$. Furthermore, a significant task $\times$ ROI interaction $(F(3,24)=11.52, P<0.005)$ indicates that this effect was strongest at posterior sides. Post-hoc comparisons between anterior and posterior regions showed higher posterior P3 amplitudes in the easy $(3.40 \mu \mathrm{V})$ as compared to the hard task $(0.75 \mu \mathrm{V} ; F(1,8)=10.75, P<0.05)$. No differences were found for anterior regions $(F(1,8)=0.23$, $P<0.7)$. An ANOVA for the target P3 in a later time interval showed higher amplitudes for the difficult $(0.92$ $\mu \mathrm{V})$ as compared to the easy task $(-0.21 \mu \mathrm{V}$; $F(3,24)=20.61, \quad P<0.005)$. A significant task $\times$ ROI interaction $(F(3,24)=6.45, P<0.005)$ further indicates that this effect was pronounced at posterior sites. Post-hoc analyses revealed enhanced late target $\mathrm{P} 3$ amplitudes for the hard task in anterior $(F(1,8)=9.31, P<0.05)$ and poster- ior regions $(F(1,8)=17.58, P<0.005)$. As for the $\mathrm{P} 3$, a significant task $\times$ ROI interaction was found for the $\mathrm{N} 2 \mathrm{~b}$ amplitudes of the targets $(F(3,24)=6.60, P<0.05)$. However, no significant differences were found between the hard and the easy task for anterior $(F(1,8)=1.17$, $P<0.4)$ and posterior $(F(1,8)=2.39, P<0.2)$ regions, indicating that $\mathrm{N} 2 \mathrm{~b}$ in targets was not enhanced in the hard task. No differences between the target amplitudes were found for the $\mathrm{N} 170(F(1,8)=1.66, P<0.3)$.

\subsection{Gamma responses}

Bursts of gamma activity after stimulus onset were only found for the evoked EEG gamma activity. The induced gamma activity did not increase after stimulus onset.

Evoked gamma activity at some selected electrodes is shown in Fig. 6 for the standard dark green circles. In all 

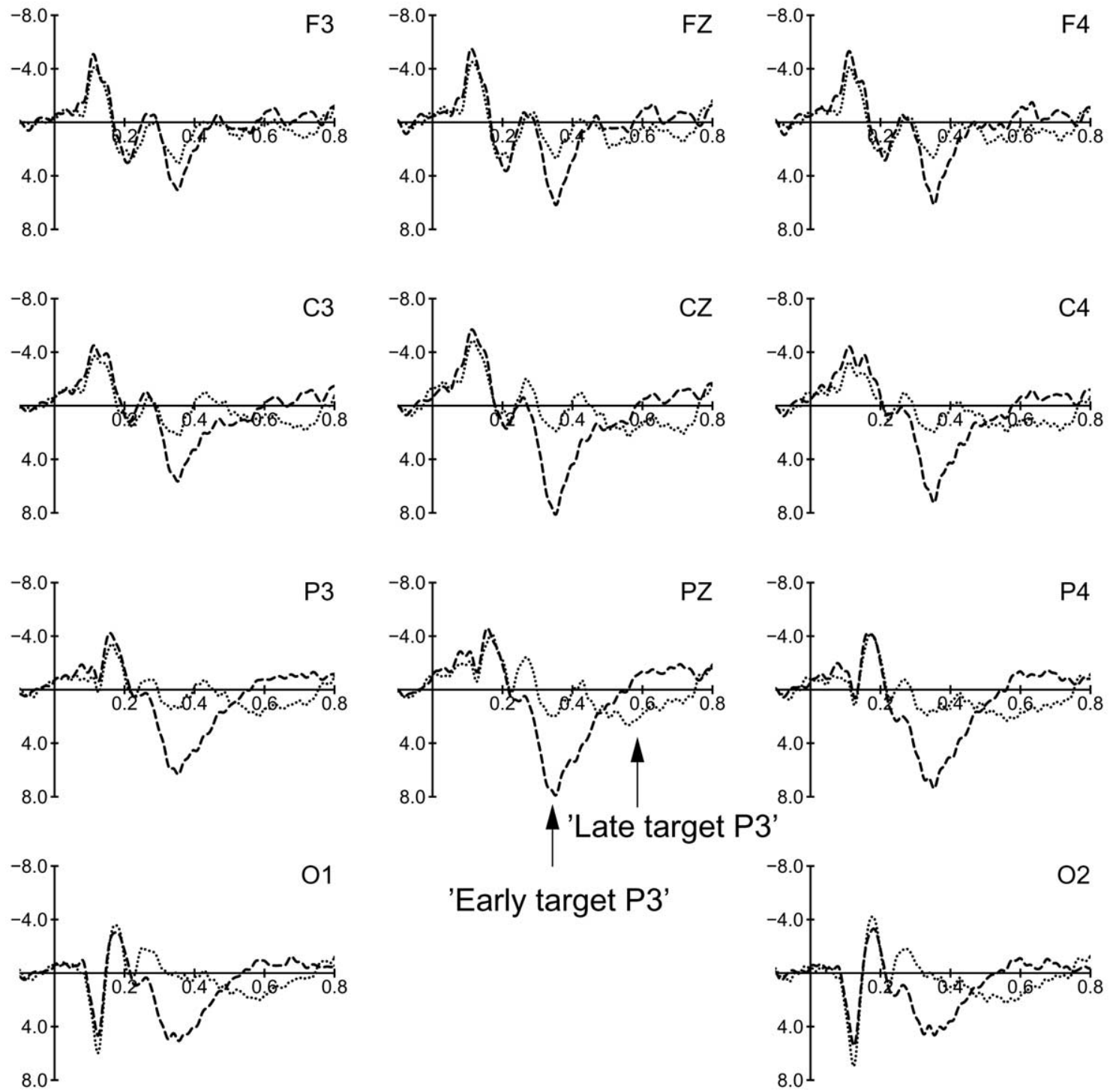

Fig. 5. ERPs averaged across 9 subjects in response to the light green target stimulus in the hard task (dotted) and target red stimulus in the easy task (dashed).

3 tasks a peak of gamma activity was found in a post-stimulus time interval between 60 and $100 \mathrm{~ms}$. An ANOVA for this time interval revealed a significant main effect of task $(F(2,16)=4.77, \quad P<0.05)$. Post-hoc comparisons revealed that the gamma activity for the dark green circle in the easy task $(0.34 \mu \mathrm{V})$ was larger than the gamma activity in the hard task $(0.28 \mu \mathrm{V} ; F(1,8)=7.13, P<0.05)$ and larger than the gamma activity in the passive task $(0.24 \mu \mathrm{V}$; $F(1,8)=11.17, P<0.05)$. A significant task $\times$ ROI interaction indicated that these differences were most pronounced over posterior electrodes $(F(3,24)=4.33$, $P<0.05)$. No gamma differences were found between the hard and the passive task in this time interval.

A second peak of gamma activity was found in the time interval between 150 and $250 \mathrm{~ms}$. An ANOVA for this time interval revealed a significant main effect of task $(F(2,16)=9.12, P<0.005)$. Largest amplitudes for this time interval were found for the hard task $(0.29 \mu \mathrm{V})$. Post-hoc comparisons revealed that the amplitudes for the hard task were larger as compared to the amplitudes for the easy task $(0.23 \mu \mathrm{V} ; F(1,8)=5.58, P<0.05)$. Furthermore, post-hoc tests also revealed larger amplitudes for the easy as compared to the passive task $(0.17 \mu \mathrm{V}$; $F(1,8)=6.38, P<0.05)$. The ANOVA for the target stimuli revealed no significant differences in evoked gamma activity.

\subsection{Latency of evoked gamma responses and P3 amplitudes}

For the examination of the relation between evoked gamma activity and P3 amplitude we correlated the latency differences of the posterior $\mathrm{Pz}$ electrode between the hard and the easy task for the EEG gamma activity (highest activity in a time range between 60 and $300 \mathrm{~ms}$ ) with the 

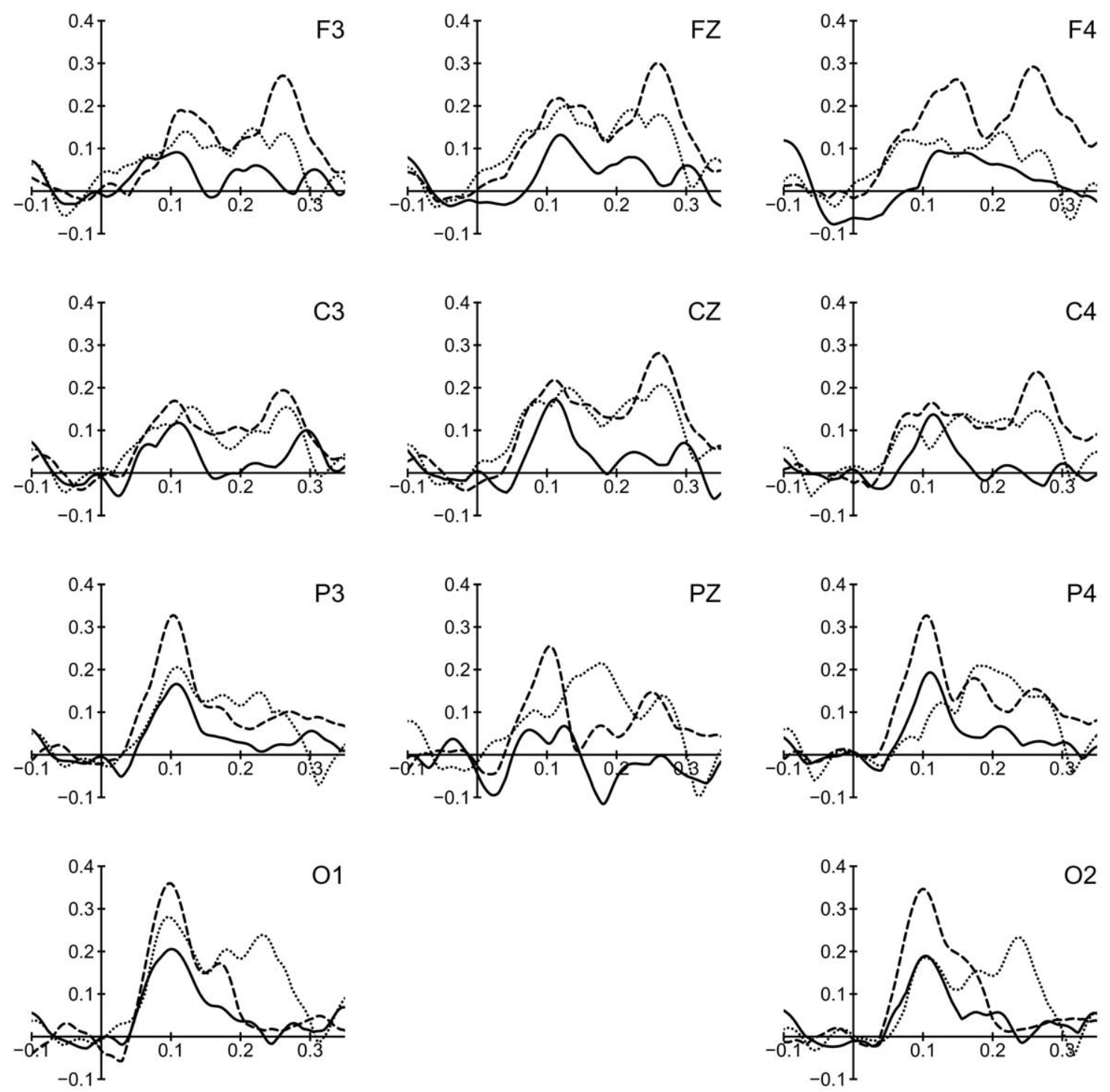

Fig. 6. Evoked EEG gamma activity averaged across 9 subjects in response to the standard dark green stimulus in the control task (solid), easy task (dashed) and hard task (dotted). Notice the second peak of gamma activity in the hard task at about 200-250 ms at posterior electrodes.

latency differences of the P3 component (highest activity in a time range between 300 and $700 \mathrm{~ms}$ ). In this analysis, a high $(r=0.53, P<0.15)$ but non-significant correlation was found. However, the lack of correlation may be explained by the small number of subjects $(n=9)$ which were included in the calculation of the correlation.

\section{Discussion}

\subsection{Behavioral data}

Higher error rates and longer RTs in the hard as compared to the easy task indicated that the hard task was in fact more difficult to perform than the easy task (Fig. 3). Longest RTs and highest error rates in both discrimination tasks were found for the target stimuli. This effect has also been reported in previous experiments (Teichner and Krebs, 1974; Herrmann and Mecklinger, 2000). A higher error rate and longer RTs for the standard dark green stimulus in the hard task further demonstrated the effect of task difficulty. Longer RTs for the dark green stimulus and the target stimulus additionally indicated a longer duration of stimulus processing in the hard task.

\subsection{ERP responses}

Our results replicated previous examinations, which found a positive relation between specific ERP components and task difficulty. Most sensitive to task difficulty was the posterior $\mathrm{N} 2 \mathrm{~b}$. The $\mathrm{N} 2 \mathrm{~b}$ component, which has its maximum amplitude at electrode $\mathrm{Cz}$ at about $260 \mathrm{~ms}$, was associated with attention to color and target detection effects (Ruijter et al., 2000; Potts and Tucker, 2001). Eimer (1996) examined 
two visual discrimination tasks with colored stimuli, where he presented targets among various distractors. He found an enhanced negativity in the time range of the $\mathrm{N} 2$ for the target, which he interpreted as a neuronal correlate of an attentional filtering process. Following this interpretation, we propose that the enhanced $\mathrm{N} 2 \mathrm{~b}$ in the hard task is a marker of the demands of a visual discrimination task. In the present study the demands differ between the two tasks. In the hard task subjects had to perform a color discrimination within one color category (light vs. dark green) and further had to produce the same response to two non-target stimuli (light green and red circle) belonging to two different categories. In particular, the discrimination within the same color category (light vs. dark green) makes the hard task more difficult than the easy task, in which a response can be made based simply on differentiating two major color categories (green and red).

Another component which was associated with task difficulty was the N170. A study which observed timing of color-based attentional processes indicates that this component was probably generated in inferior occipito-temporal cortex (Anllo-Vento et al., 1998). Vogel and Luck (2000) reported an enhanced negative deflection of the N170 component in choice-RT tasks with colorful letter arrays. In their study the influence of task difficulty by varying the distractor colors has been investigated in an easy and in a hard choice-RT task. The target in both tasks was a nonspecific red letter. Colors of the distractor letters could either be blue, gray, green or violet (easy task), or shades of purple and pink (hard task). Compared to the simple-RT task, the N170 was increased in both choice-RT tasks. No differences in the time range of the N170 component were found between the easy and hard task. The present study replicated this finding, which indicates that N170 is generally enhanced in visual discrimination processes.

Amplitude differences in the study of Vogel and Luck (2000) were also observed for the time interval of the P3 component. It was suggested that more difficult discriminations tend to produce smaller and later P3s (Polich and Bondurant, 1997; Grillon et al., 1990). In the present study, P3 was also delayed in the hard task as compared to the easy task (Fig. 5). This effect was primarily found at posterior electrodes, which is in line with previous ERP findings (Mangun et al., 1998; Karayanidis and Michie, 1997). The observed delay of $P 3$ amplitude could indicate a longer duration of stimulus evaluation (Donchin and Coles, 1988). This assumption was supported by higher RTs in the hard task.

\subsection{Gamma responses}

The main purpose of this study was to investigate the relation between the difficulty of visual discrimination tasks and EEG gamma activity. A higher evoked EEG gamma activity was found in two color discrimination tasks as compared to a passive control task. Evoked gamma activity in an easy discrimination task was found in a time range of about $100 \mathrm{~ms}$. In a hard discrimination task, evoked gamma activity was found in a later time range of about $200 \mathrm{~ms}$. The delay of gamma activity suggests a longer duration of stimulus processing in the hard discrimination task. This interpretation fits well with the assumption of a relation between EEG gamma activity and the processing demands of a task (Simos et al., 2002; Yordanova et al., 1997b). Simos et al. (2002) examined the influence of task complexity on EEG gamma activity by manipulating the complexity of a target stimulus which had to be detected. In an easy task, subjects had to decide whether they saw a specific animal (a dog or a cat) after the presentation of different animal pictures. In more complex tasks, subjects had to detect a stimulus with specific features (e.g. a 4legged animal among the animal pictures). The authors reported a linear increase in absolute power in the gamma band over right temporoparietal, left occipital, and left frontal regions with increasing task complexity. These regions also showed a relation with task difficulty in the present study, where strongest gamma effects were found at posterior sites (Fig. 6). Posterior gamma activity has been associated with attentional processes in selective visual attention (Fries et al., 2001), in a paired stimuli paradigm (Shibata et al., 1999a) and in target detection (Herrmann et al., 1999). Clinical studies (Miceli et al., 2001; Schoppig et al., 1999), PET examinations (Gulyas and Roland, 1991), fMRI studies (Engel et al., 1997) and animal studies (Hanazawa et al., 2000; Johnson et al., 2001) further showed that different posterior regions like visual cortex, inferior temporal lobe and left superior parietal cortex are crucially involved in visual discrimination processes. We therefore suggest that the delayed posterior gamma activity is related to such discrimination processes.

The finding that only the evoked but not the induced gamma activity was related to the onset of an experimental condition goes well in line with our previous EEG and MEG examinations in visual discrimination tasks (Herrmann et al., 1999; Herrmann and Mecklinger, 2000). In these studies, we also failed to find induced gamma activity related to the experimental conditions. However, an enhanced induced gamma activity has been found in cognitive processes like visual short-term memory (TallonBaudry et al., 1998) and learning processes (Gruber et al., 2001). Yordanova et al. (1997a) and Fell et al. (1997) reported a higher degree of phase-locking to targets than to standard stimuli, indicating that task difficulty may alter gamma phase-locking. This may be the reason why we found only evoked but no induced gamma responses: since both color discrimination tasks are at least as hard as a simple target discrimination task, both lead to phaselocked gamma responses.

\subsection{General discussion}

When interpreting the results with relation to task diffi- 
culty one has to keep in mind that the easy and the hard task require different kinds of processing. In the easy task, subjects had to perform a simple color category comparison between the target red and the two non-target green circles. Similar RTs and error rates (Fig. 3) indicate that the two standard stimuli (light and dark green circles) were both processed in a similar way in the easy task. This result contrasts with that found for the hard task, where higher RTs and a higher error rate were found for the dark green circle as compared to the red circle. To execute the hard task, a comparison of two color categories is required. For the detection of the light green circle subjects had to perform a discrimination within the same color category (light green vs. dark green). This discrimination makes the task more difficult to perform. In addition to discrimination within one color category the same response to different color categories (red and green) has to be executed for the two non-target stimuli. This raises the following question: which differences on the behavioral side are most relevant in explaining the presented data?

One factor which might be related to the EEG gamma effects is the different RT of the easy (408 ms) and the hard task $(570 \mathrm{~ms})$. Could the delayed motor responses in the hard task be an explanation for the delayed EEG gamma activity? Crone et al. (1998) examined event-related synchronization for the gamma band of the sensorimotor cortex with subdural electrocorticographic electrodes. They found that gamma synchronization began slightly before or during the motor response. The gamma effects (60-250 ms) in the present study were found long before the motor response of the subjects. It is therefore unlikely that different latencies of the motor responses could explain these effects.

Another aspect which might explain the delayed gamma activity in the hard task is a possible inhibition of a target response in the hard task. The two green circles had a very similar color and were therefore difficult to discriminate. For this reason, we propose that the processing of the nontarget green stimulus in the hard task requires an inhibition of a target response. Longer RTs and a higher error rate in the hard task for the non-target dark green stimulus as compared to the non-target red stimulus may be indicative of this inhibition of the target response.

The inhibition of a target response has been examined extensively in the go/nogo paradigm where a target response has to be suppressed (Pfefferbaum et al., 1985). In go/nogo studies, an enhanced frontocentral N2 can be observed for the nogo trials (Bokura et al., 2001; Jackson et al., 1999). In the present study the $\mathrm{N} 2$ for the non-target dark green stimulus was also enhanced in the hard task as compared to the easy task in frontal regions. This could indicate the inhibition of a target response for the non-target green stimulus. However, the strongest N2 differences were found at posterior sites and not at frontal sites (Fig. 4), indicating that the enhanced posterior $\mathrm{N} 2$ in the hard task is directly related to the difficulty of visual discrimination tasks. Inhibition of behavior has also been related to the relation to EEG gamma activity (Shibata et al., 1999b). In that study, an enhanced gamma band oscillation was found in central regions $(\mathrm{C} 3, \mathrm{C} 4$ and $\mathrm{Cz})$ in a time range at around 230 ms. Interestingly, this is the same time range where an enhanced gamma activity for the hard task was found (Fig. 6), indicating that the enhanced gamma activity in the hard task might be explained by an inhibition of a target response. However, since target inhibition processes have been primarily related to anterior regions, we argue that these processes cannot explain the posterior gamma effects. We therefore concluded that the delayed posterior evoked gamma activity indicates a longer duration of the discrimination process due to the additional complexity of the hard task (stimulus discrimination within one color category and response discrimination across color categories).

Interestingly, gamma activity reveals the difference in task difficulty within $100 \mathrm{~ms}$ whereas ERPs only reflect this difference after about $260 \mathrm{~ms}$. We therefore propose that oscillatory EEG activity may be a better indicator of task difficulty than ERPs are. There are remarkable similarities between evoked gamma activity and ERP data with respect to task difficulty. Evoked gamma activity of the standard dark green stimulus showed a delay of about 100 $\mathrm{ms}$ in the hard task (Fig. 6). This delay of gamma activity was similar to the delayed latency of the P3 component in ERPs (Fig. 5). We suggest that the delayed P3 and evoked gamma activity both reflect a longer duration of stimulus processing. This would indicate a close functional relation between evoked gamma activity and the P3 component. A high but non-significant correlation between the latency differences (hard task vs. easy task) of the P3 amplitude and evoked gamma activity is consistent with this conclusion. The assumption of a close relation between oscillatory processes and ERP components was also strongly supported by recently published data (Başar et al., 2001; Makeig et al., 2002). However, further investigations are necessary to examine the functional relations between ERP components and EEG gamma activity.

\section{Acknowledgements}

We express our thanks to Heiko Temming for his technical assistance, to Axel Mecklinger for his insightful comments on the manuscript and Ulrike Barth who helped during data acquisition. Daniel Senkowski was funded by the German Research Foundation (DFG, grant HE 3353/1). This study was supported by the Max Planck Society.

\section{References}

Anllo-Vento L, Luck SJ, Hillyard SA. Spatio-temporal dynamics of attention to color: evidence from human electrophysiology. Hum Brain Mapp 1998;6:216-238.

Başar E, Schürmann M, Demiralp T, Başar-Eroglu C, Ademoglu A. Event- 
related oscillations are 'real brain responses' - wavelet analysis and new strategies. Int J Psychophysiol 2001;39:91-127.

Bokura H, Yamaguchi S, Kobayashi S. Electrophysiological correlates for response inhibition in a Go/NoGo task. Clin Neurophysiol 2001;112:2224-2232.

Breton F, Ritter W, Simson R, Vaughan Jr. HG. The N2 component elicited by stimulus matches and multiple targets. Biol Psychol 1988;27:23-44.

Comerchero MD, Polich J. P3a and P3b from typical auditory and visual stimuli. Clin Neurophysiol 1999;110:24-30.

Crone NE, Miglioretti DL, Gordon B, Lesser RP. Functional mapping of human sensori-motor cortex with electrocorticographic spectral analysis. II. Event-related synchronization in the gamma band. Brain 1998;121:2301-2315.

De Pascalis V, Ray WJ. Effects of memory load on event-related patterns of 40-Hz EEG during cognitive and motor tasks. Int J Psychophysiol 1998;28:301-315.

Donchin E, Coles MG. Is the P300 component a manifestation of context updating?. Behav Brain Sci 1988;11:357-374.

Eimer M. The N2pc component as an indicator of attentional selectivity. Electroenceph clin Neurophysiol 1996;99:225-234.

Elliott MA, Herrmann CS, Mecklinger A, Muller HJ. The loci of oscillatory visual-object priming: a combined electroencephalographic and reaction-time study. Int J Psychophysiol 2000;38:225-241.

Engel AK, Singer W. Temporal binding and the neural correlates of sensory awareness. Trends Cogn Sci 2001;5:16-25.

Engel S, Zhang X, Wandell B. Colour tuning in human visual cortex measured with functional magnetic resonance imaging. Nature 1997;388:68-71.

Fell J, Hinrichs H, Roschke J. Time course of human $40 \mathrm{~Hz}$ EEG activity accompanying $\mathrm{p} 3$ responses in an auditory oddball paradigm. Neurosci Lett 1997;235:121-124.

Fell J, Klaver P, Lehnertz K, Grunwald T, Schaller C, Elger CE, Fernandez G. Human memory formation is accompanied by rhinal-hippocampal coupling and decoupling. Nat Neurosci 2001;4:1259-1264.

Fries P, Reynolds JH, Rorie AE, Desimone R. Modulation of oscillatory neuronal synchronization by selective visual attention. Science 2001;291:1560-1563.

Galambos R. A comparison of certain gamma band $(40 \mathrm{~Hz})$ brain rhythms in cat and man. In: Başar E, Bullock T, editors. Induced rhythms in the brain, Boston, MA: Birkhäuser, 1992. pp. 201-216.

Grillon C, Courchesne E, Ameli R, Elmasian R, Braff D. Effects of rare non-target stimuli on brain electrophysiological activity and performance. Int J Psychophysiol 1990;9:257-267.

Gruber T, Keil A, Muller MM. Modulation of induced gamma band responses and phase synchrony in a paired associate learning task in the human EEG. Neurosci Lett 2001;316:29-32.

Gulyas B, Roland PE. Cortical fields participating in form and colour discrimination in the human brain. NeuroReport 1991;2:585-588.

Hanazawa A, Komatsu H, Murakami I. Neural selectivity for hue and saturation of colour in the primary visual cortex of the monkey. Eur J Neurosci 2000;12:1753-1763.

Herrmann CS, Knight RT. Mechanisms of human attention: event-related potentials and oscillations. Neurosci Biobehav Rev 2001;25:465-476.

Herrmann CS, Mecklinger A. Magnetoencephalographic responses to illusory figures: early evoked gamma is affected by processing of stimulus features. Int J Psychophysiol 2000;38:265-281.

Herrmann CS, Mecklinger A. Gamma activity in human EEG is related to high-speed memory comparisons during object selective attention. Vis Cogn 2001;8:593-608.

Herrmann CS, Mecklinger A, Pfeifer E. Gamma responses and ERPs in a visual classification task. Clin Neurophysiol 1999;110:636-642.

Hoffman JE, Houck MR, MacMillan 3rd FW, Simons RF, Oatman LC. Event-related potentials elicited by automatic targets: a dual-task analysis. J Exp Psychol Hum Percept Perform 1985;11:50-61.

Jackson SR, Jackson GM, Roberts MR. The selection and suppression of action: ERP correlates of executive control in humans. NeuroReport 1999;10:861-865
Johnson EN, Hawken MJ, Shapley R. The spatial transformation of color in the primary visual cortex of the macaque monkey. Nat Neurosci 2001;4:409-416.

Karayanidis F, Michie PT. Evidence of visual processing negativity with attention to orientation and color in central space. Electroenceph clin Neurophysiol 1997;103:282-297.

Keil A, Müller MM, Gruber T, Wienbruch C, Stolarova M, Elbert T. Effects of emotional arousal in the cerebral hemispheres: a study of oscillatory brain activity and event-related potentials. Clin Neurophysiol 2001;112:2057-2068.

Krüger J, Lamme VAF, Bondar I. Colour-related oscillations in the striate cortex of awake monkeys: "reverse" observations. Brain Topogr 2002;14:191-196.

Makeig S, Westerfield M, Jung TP, Enghoff S, Townsend J, Courchesne E, Sejnowski TJ. Dynamic brain sources of visual evoked responses. Science 2002;295:690-694.

Mangun GR, Buonocore MH, Girelli M, Jha AP. ERP and fMRI measures of visual spatial selective attention. Hum Brain Mapp 1998;6:383389.

Mecklinger A, Maess B, Opitz B, Pfeifer E, Cheyne D, Weinberg H. A MEG analysis of the P300 in visual discrimination tasks. Electroenceph clin Neurophysiol 1998;108:45-56.

Miceli G, Fouch E, Capasso R, Shelton JR, Tomaiuolo F, Caramazza A. The dissociation of color from form and function knowledge. Nat Neurosci 2001;4:662-667.

Müller MM, Keil A, Gruber T, Elbert T. Processing of affective pictures modulates right-hemispheric gamma band EEG activity. Clin Neurophysiol 1999;110:1913-1920.

Müller MM, Gruber T, Keil A. Modulation of induced gamma band activity in the human EEG by attention and visual information processing. Int $\mathrm{J}$ Psychophysiol 2000;38:283-299.

Näätänen R, Gaillard AW, Mantysalo S. Early selective-attention effect on evoked potential reinterpreted. Acta Psychol Amst 1978;42:313329.

Palmer B, Nasman VT, Wilson GF. Task decision difficulty: effects on ERPs in a same-different letter classification task. Biol Psychol 1994;38:199-214.

Pfefferbaum A, Ford JM, Weller BJ, Kopell BS. Erps to response production and inhibition. Electroenceph clin Neurophysiol 1985;60:423434.

Polich J. Task difficulty, probability, and inter-stimulus interval as determinants of P300 from auditory stimuli. Electroenceph clin Neurophysiol 1987;68:311-320.

Polich J, Bondurant T. P300 sequence effects, probability, and interstimulus interval. Physiol Behav 1997;61:843-849.

Potts GF, Tucker DM. Frontal evaluation and posterior representation in target detection. Brain Res Cogn Brain Res 2001;11:147-156.

Ritter W, Simson R, Vaughan Jr HG, Macht M. Manipulation of eventrelated potential manifestations of information processing stages. Science 1982;218:909-911.

Ritter W, Simson R, Vaughan Jr HG. Event-related potential correlates of two stages of information processing in physical and semantic discrimination tasks. Psychophysiology 1983;20:168-179.

Ritter W, Simson R, Vaughan Jr HG. Effects of the amount of stimulus information processed on negative event-related potentials. Electroenceph clin Neurophysiol 1988;69:244-258.

Ruijter J, de Ruiter MB, Snel J, Lorist MM. The influence of caffeine on spatial-selective attention: an event-related potential study. Clin Neurophysiol 2000;111:2223-2233.

Schoppig A, Clarke S, Walsh V, Assal G, Meuli R, Cowey A. Short-term memory for colour following posterior hemispheric lesions in man. NeuroReport 1999;10:1379-1384.

Shibata T, Shimoyama I, Ito T, Abla D, Iwasa H, Koseki K, Yamanouchi N, Sato T, Nakajima Y. Attention changes the peak latency of the visual gamma-band oscillation of the EEG. NeuroReport 1999a;10:11671170.

Shibata T, Shimoyama I, Ito T, Abla D, Iwasa H, Koseki K, Yamanouchi N, 
Sato T, Nakajima Y. Event-related dynamics of the gamma-band oscillation in the human brain: information processing during a go/nogo hand movement task. Neurosci Res 1999b;33:215-222.

Simos PG, Papanikolaou E, Sakkalis E, Micheloyannis S. Modulation of gamma-band spectral power by cognitive task complexity. Brain Topogr 2002;14:191-196.

Smid HG, Jakob A, Heinze HJ. An event-related brain potential study of visual selective attention to conjunctions of color and shape. Psychophysiology 1999;36:264-279.

Tallon C, Bertrand O, Bouchet P, Pernier J. Gamma-range activity evoked by coherent visual stimuli in humans. Eur J Neurosci 1995;7:1285-1291.

Tallon-Baudry C, Bertrand O, Peronnet F, Pernier J. Induced gamma-band activity during the delay of a visual short-term memory task in humans. J Neurosci 1998;18:4244-4254.

Teichner WH, Krebs MJ. Laws of visual choice reaction time. Psychol Rev 1974;81:75-98.

Vogel EK, Luck SJ. The visual N1 component as an index of a discrimination process. Psychophysiology 2000;37:190-203.

Yordanova J, Kolev V, Demiralp T. Effects of task variables on the amplitude and phase-locking of auditory gamma band responses in human. Int J Neurosci 1997a;92:241-258.

Yordanova J, Kolev V, Demiralp T. The phase-locking of auditory gamma band responses in humans is sensitive to task processing. NeuroReport 1997b;8:3999-4004. 